\title{
IDEE IN DISCUSSIONE
}

\section{LA REGIONE VENETO INCONTRA LE POLICIES EUROPEE}

\section{Recensione a Messina P., a cura di, Innovare la tradizione ${ }^{*}$}

Quanto incidono le politiche europee sulle istituzioni e sulle politiche nazionali? Come misurare tale impatto e quali fattori favoriscono o, al contrario, impediscono il cambiamento nei vari domini della vita pubblica ( $p o-$ litics, policies, polity) degli Stati membri a seguito dell'adeguamento agli imperativi formulati dall'Unione Europea (Ue)?

Da qualche decennio, ormai, gli studi sull'europeizzazione, incentrati per l'appunto sull'interazione delle arene politiche nazionali con quella europea, cercano di rispondere alle summenzionate domande concernenti il processo di trasformazione. Esso, talvolta, comporta modifiche dei sistemi di governance domestici attraverso la diffusione di costruzioni ideazionali, norme legali e sociali, regolazioni e strumenti, i quali sono dapprima identificati ed istituzionalizzati all'interno delle arene politico-decisionali dell'Ue, e poi eventualmente utilizzati dagli attori domestici per modificare i propri ordini istituzionali e/o comportamentali.

Facendo tesoro di un'esperienza di collaborazione con le istituzioni regionali e di ricerca pluriennale sul tema dell'europeizzazione, il libro curato da P. Messina illustra in modo dettagliato come il processo in questione abbia contribuito alla trasformazione del modo di regolazione della Regione Veneto, confrontando l'impatto che ha avuto l'Ue sugli equilibri tradizionali in questa Regione con gli scenari verificatesi in altri contesti regionali italiani e stranieri.

L'analisi empirica presentata nei capitoli che trattano i diversi risvolti dell'europeizzazione, è introdotta da un capitolo teorico-analitico di S. Dossi e C. Radaelli), che apre il volume con una riflessione sulle possibili definizioni, interpretazioni e strumenti di analisi di questo processo, dedicando particolare attenzione alle dimensioni analitiche che possono essere impiegate a tale scopo e, in particolare, per quanto concerne le Regioni. La proposta metodologica avanzata dagli autori, basata su una ragionata

\footnotetext{
* Patrizia Messina, a cura di, (2011), Innovare la tradizione. Europeizzazione e governance regionale: il caso del Veneto a confronto, Cleup, Padova (pp. 248, € 16,00).
} 
rassegna della letteratura sul tema, suggerisce di considerare le Regioni come "spazi politici" caratterizzati da specifiche strutture di organizzazione dei poteri, da logiche d'azione peculiari e da relazioni tra attori che agiscono in sistemi territoriali diversi. L'analisi dell'europeizzazione richiederebbe, dunque, di rivolgere l'attenzione di ricerca verso le differenti arene di policy che coinvolgono le regioni in Europa, e cercare di mettere in luce le varie costellazioni degli attori e i processi di interazione fra loro.

Proseguendo nella direzione appena delineata, il capitolo di P. Messina e C. Busatto spiega come il modo di regolazione tradizionale della Regione Veneto si stia modificando sotto la pressione regolativa europea. Le trasformazioni rilevate dalla ricerca concernono sia l'assetto organizzativo dell'Ente regionale, sia lo stile amministrativo e i modi di policy-making. Viene valutato, dunque, l'impatto dell'Ue come un fattore esterno di innovazione, che porta le istituzioni domestiche ad adeguare la propria struttura e l'operato alle esigenze legate all'implementazione delle politiche comunitarie. Tale impatto risulta particolarmente rilevante nell'ambito di gestione dei fondi strutturali che, oltre ai cambiamenti strutturali nell'organizzazione dell'amministrazione regionale, ha richiesto il cambiamento dello stile di governo, verso l'adempimento dei principi cardine della politica di coesione europea, ovvero la programmazione, il partenariato, la concentrazione, ecc. Le conclusioni del capitolo sottolineano come la sfida europea non sia solo di tipo amministrativo, ma essenzialmente di tipo regolativo e culturale, mentre la variabile politica gioca un ruolo fondamentale nella definizione della direzione e dell'intensità del processo di trasformazione.

Tale quadro viene ulteriormente articolato da F. Zecchetto, che illustra i limiti e i successi della Regione Veneto nello sfruttare le opportunità offerte dalla politica di coesione europea, sia in termini di risorse finanziarie che dal punto di vista di partecipazione ai canali istituzionali che collegano le arene politiche regionali con le istituzioni europee. Dal saggio di Zecchetto si evince un dinamico e tempestivo adeguamento della Regione alle regole europee sotto il profilo istituzionale, mentre nella trasformazione dello stile di policy rimangono ampi margini di miglioramento e approfondimento, soprattutto in termini di diffusione orizzontale di principi e di prassi amministrative coerenti con le indicazioni europee.

Una tendenza simile viene rilevata dall'analisi di A. Prontera che, ricostruendo l'impatto della stessa policy comunitaria sull'Ente regionale e sullo stile di policy delle Marche, evidenzia alcuni nodi spinosi nel processo di adeguamento alle regole e alle norme dell'Ue. Si sottolinea in particolare la difficoltà di superare la fase del cosiddetto adattamento, che consiste nell'adempimento formale delle richieste dell'Ue, passando ad una fase 
qualitativamente superiore di apprendimento che comporta la trasformazione vera e propria.

La ricostruzione delle trasformazioni avvenute nell'ambito di altre politiche pubbliche, tra cui lo sviluppo sostenibile, lo sviluppo rurale, le piccole e medie imprese e l'immigrazione, mette in luce quando sia stato farraginoso e difficoltoso il processo di europeizzazione nella Regione Veneto, anche in confronto ad altre esperienze regionali.

In particolare, ricostruendo il processo di adeguamento regionale alla strategia europea dello sviluppo sostenibile, il capitolo di G. Vanin illustra uno squilibrio tra la dimensione formale e quella sostanziale. La Regione Veneto ha adempiuto infatti agli obblighi normativi comunitari in materia; al tempo stesso però, nella percezione delle élite politiche e amministrative, appare carente la conoscenza delle priorità della strategia europea in questione nonché la coscienza della sua importanza nelle politiche comunitarie e quelle regionali nel loro insieme.

L'indagine di E. Gottardo, che esplora le politiche per lo sviluppo rurale della Regione Veneto a confronto con quelle della Regione South West England concentrandosi sul grado di apprendimento sociale e sul cambiamento dei paradigmi politici, sottolinea come i percorsi sviluppati dal basso, che uniscono le comunità locali attorno ad un progetto condiviso di sviluppo, possano fare la differenza in termini di competitività e sostenibilità dei territori. Alla luce di questo, suggerisce l'autrice, la funzione della regolazione politica dovrebbe consistere in un'attenta lettura e programmazione delle azioni per un territorio, incentrata su intersettorialità e dinamicità per la produzione di beni pubblici locali.

Differenze nel processo di europeizzazione delle Regioni emergono anche dallo studio delle strategie che esse mettono in atto per collegare le arene politiche regionali con quella europea. Come illustra il contributo di R. Nardo, infatti, le tre Regioni messe a confronto si caratterizzano per diversi stili di rappresentanza. Alcune, come la Regione Veneto, tendono a promuovere gli interessi istituzionali, talvolta a discapito della funzione di sensibilizzazione e di attivazione degli attori locali nell'arena domestica verso quella europea. Altre Regioni, tra cui l'Emilia Romagna, hanno una forte vocazione di costruzione di reti, sia nell'ambito delle arene locali, sia a livello europeo, con le regioni di altri paesi. Questo dimostra quanto la cultura di governo e lo stile amministrativo possano essere determinanti per la modalità di attivazione di una Regione nell'arena politica comunitaria.

Il volume fornisce, dunque, un ricco quadro per la riflessione sulla trasformazione che le istituzioni pubbliche hanno attraversato negli ultimi decenni, mettendo in risalto le sfide che il processo della progressiva espan- 
sione della regolazione europea ha prodotto in termini di cambiamento istituzionale e di policy a livello sub-statale. Nel trarre le conclusioni finali, il capitolo di P. Messina evidenzia come non si tratti di uno scenario convergente essendovi una diversa capacità di adattamento e di adeguamento alle direttive europee nei vari Stati e Regioni. Sul grado di cambiamento che avviene a seguito della spinta all'innovazione derivante dall'Europa incidono, infatti, alcune variabili interne alle arene politiche domestiche e, in particolare, i modi di governance delle arene politiche domestiche, lo stile amministrativo e lo stile di policy esistente. In definitiva, i contributi raccolti nel volume dimostrano chiaramente come l'europeizzazione sia un processo di trasformazione complesso e articolato, di forte vocazione culturale, che coinvolge e mette alla prova dimensioni politiche chiave, quali modelli di rappresentanza, modalità decisionali, stili di policy e di partecipazione.

Ekaterina Domorenok 